netopause to the surface of the neutron star results in the observed oscillations of the rapid burster. The fact that the hardness ratios of the bursts at the maximum and the minimum of the pulse are the same supports this possibility.

We thank the crew of the Hakucho satellite for providing observational data and to Professors Y. Tanaka and $\mathrm{M}$. Matsuoka for their valuable comments.

Received 18 February; accepted 23 June 1982

. Kunieda, H. et al. Publ. astr. Soc. Japan (in the press)

2. Inoue, $H$. et al. Nature 283, $358(1980)$

. Lewin, W. H. G. et al. Astrophys. J. Lett. 207, L95 (1976)

. Cordova, F. A., Chester, T. J., Tuohy, I. R. \& Garmire, G. P. Astrophys. J. 235, 163 (1980)

Patterson, J. Astrophys. J. Suppl, 45, 517 (1981).

6. Van Horn, H. M., Wesemael, F. \& Winget, D. E. Astrophys. J. Lett. 235, L143 (1980).

\section{A possible resurfacing mechanism for icy satellites}

\section{J. Klinger}

Laboratoire de Glaciologie et Géophysique de l'Environnement, 38031 Grenoble Cedex, France

Photographs from Voyager Missions reveal a great variety of surface features for the icy satellites of Saturn'. Mimas is covered with many old craters. Enceladus has a rather smooth surface with few and rather young craters. Some parts of Dione's surface have an important crater density, whereas other parts are relatively smooth. Thus some of Saturn's icy satellites seem to have undergone important surface modifications during their history while others did not. I suggest here that the irreversible phase transition between amorphous and cubic ice and similar phase transitions may provide a low energy resurfacing mechanism for these icy satellites.

To explain the resurfacing of Enceladus it has been suggested ${ }^{2}$ that its interior may contain liquid water covered only by a thin crust of ice. For a given thickness of the crust, it is possible to estimate what the subsurface heat flux must be. For a surface temperature of $100 \mathrm{~K}$, a liquid core temperature of $273 \mathrm{~K}$ and a heat conduction coefficient like that of normal hexagonal ice ${ }^{3}$ we obtain a heat flux in the order of magnitude of $0.1 \mathrm{~W} \mathrm{~m}^{-2}$. This is comparable to the mean terrestrial heat flux $\left(\sim 0.063 \mathrm{~W} \mathrm{~m}^{-2}\right)$. If this heat flux has to be maintained permanently by tidal heating, the tidal dissipation must be at least $7 \times 10^{10} \mathrm{~W}$ in the whole satellite. Peale et al. ${ }^{4}$ calculated the average volumetric tidal dissipation in Saturn's satellites. They found for Enceladus $2.2 \times 10^{-10} \mathrm{~W} \mathrm{~m}^{-3}$. That corresponds to a total dissipation of $1.4 \times 10^{7} \mathrm{~W}$. So it seems difficult to maintain a liquid core by tidal heating and even more difficult to heat up a low temperature core and to melt it.

I now propose an alternative resurfacing mechanism that needs much less initial energy input. It has been shown ${ }^{3,5,6}$ that the irreversible phase transition of water ice that occurs between amorphous and cubic ice at $\sim 153 \mathrm{~K}$ may influence the heat balance of comet nuclei. The same phase transition may in some cases be responsible for resurfacing of icy satellites. Suppose that the satellite has been condensed at temperatures sufficiently low so that the ice is in the amorphous state. As amorphous ice is a very poor heat conductor, we can assume that a large part of the heat dissipated in the interior of the icy body is used to heat it up. When in one part of the satellite the transition temperature is reached, the phase transition will start. The heat release during crystallization is about $100 \mathrm{~J} \mathrm{~g}^{-1}$ (ref. 7). This energy can be used to heat the neighbourhood. On the ohter hand the heat conduction coefficient of crystalline ice at the transition temperature seems to be about 10 times higher than that of amorphous ice ${ }^{3}$ and a growing part of the internally dissipated heat will be lost to the surface. These two mechanisms are in competition. But as the crystallization of the amorphous ice will start in the interior the heat loss will be unimportant until the surface layers undergo the phase transition.

In the case of Enceladus the tidal dissipation calculated by Peale et al. ${ }^{4}$ is able to heat this body from 100 to $153 \mathrm{~K}$ in 7,000 Myr. This is evidently not realistic. But Ghormley ${ }^{7}$ found that amorphous ice condensed at $20 \mathrm{~K}$ and released $33 \mathrm{~W} \mathrm{~g}^{-1}$ during heating from 20 to $77 \mathrm{~K}$ and $92 \mathrm{~W} \mathrm{~g}^{-1}$ between 77 and $153 \mathrm{~K}$. The heat release is smaller for ice condensed at higher temperatures. This heat will considerably speed up the process and only slight heating by tidal dissipation is necessary to initiate the temperature rise.

On the other hand, Yoder ${ }^{8}$ proposed that the eccentricity of Enceladus has been pumped to a higher value than the present one. This may produce some excess heating and allow a still more rapid heating from the orbital equilibrium temperature to the phase transition temperature. Evidently the mechanism described here may be combined with resurfacing mechanisms of very different nature.

Amorphous ice has a density between 1.1 and $0.94 \mathrm{~g} \mathrm{~cm}^{-3}$ depending on the condensation temperature ${ }^{5}$. During the phase transition nearly all water molecules are reoriented and the density decreases. This decrease in density may produce surface cracks and contribute to the smoothing of existing surface features.

If the heat transport to the surface due to conduction is not able to evacuate all the heat dissipated in the icy body, the internal temperature will continue to rise and might reach the transition temperature between cubic and hexagonal ice where a second resurfacing process may take place. A third resurfacing may take place when the melting temperature of hexagonal ice can be reached. When the heat loss to the surface becomes more important than the internal dissipation the satellite will cool down to the equilibrium temperature; in the case of Saturn's satellites to a temperature near $100 \mathrm{~K}$. This cooling can take place after a partial or a complete resurfacing or after one or more resurfacing periods.

It is evident that similar mechanisms may apply when other materials undergo similar phase transitions. We know, for example, that the heat conduction coefficient of at least one clathrate hydrate is of the same order of magnitude and has a similar temperature dependence as amorphous substances ${ }^{9}$. We therefore suppose that such kind of effects will appear when clathrate hydrates reach their limits of stability.

According to Gaffney and Matson ${ }^{10}$, high pressure forms of ice may locally be produced during crater formation. Those forms are metastable at low pressures for temperatures that can be found on Jupiter's and Saturn's satellites. The lifetime of those polymorphes is evidently temperature dependent. Destruction of those ices may also contribute to a resurfacing. Gaffney and Matson suggest that icy satellites should be investigated by means of IR reflection spectroscopy. In fact, morphological studies of impact crater and other surface features combined with IR scanning reflection spectroscopy during future space missions could yield information about the chemical nature and the physical state of surface materials, on the chronology of surface smoothing, and the mechanisms involved. Those kinds of mechanism may occur on many satellites of the outer planets.

More quantitative work on the problem of the geological activity of icy satellites is planned in our laboratory. This work has been sponsored by INAG-ATP Planetologie grant 37-86.

\footnotetext{
Received 5 April; accepted 23 June 1982.

Smith, B. A. et al Science 212, 163-190 (1981)

2. Mitchell Waldrop, M. Science 213, 1236-1240 (1981).

3. Klinger,J. Science 209, 271-272 (1980).

4. Peale, S. J., Cassen, P. \& Reynolds, R. T. Icarus 43, 65-72 (1980).

5. Smoluchowski, R. Astrophys. J. Lett. 244, L31-L34 (1981).

6. Klinger, J. Icarus 47, 320-324 (1981).

7. Ghormley, J. A. J chem. Phys. 48,503-508 (1968).

8. Yoder, C. F. Nature 279, 767-770 (1979)

9. Ross, R. G., Anderson, P. \& Bäckström, Nature 290, 322-323 (1981).

10. Gaffney, E. S. \& Matson, D. L. Icarus 44, 511-519 (1980).
} 